\title{
Compatibility approach for the improvement of oxide thermoelectric converters for industrial heat recovery applications
}

\author{
Gesine Saucke, ${ }^{1}$ Sascha Populoh, ${ }^{1, a)}$ Philipp Thiel, ${ }^{1}$ Wenjie Xie, ${ }^{2}$ Ryoji Funahashi, ${ }^{3}$ \\ and Anke Weidenkaff ${ }^{2}$ \\ ${ }^{1}$ Laboratory Materials for Energy Conversion, Empa, Swiss Federal Laboratories for Materials Science \\ and Technology, Überlandstr. 129, CH-8600 Dübendorf, Switzerland \\ ${ }^{2}$ Materials Chemistry, Institute for Materials Science, University of Stuttgart, Heisenbergstr. 3 , \\ 70569 Stuttgart, Germany \\ ${ }^{3}$ Synthetic Nano-Function Materials, UBIQEN, AIST, 1-8-31 Midorigaoka, Ikeda, Osaka 563-8577, Japan
}

(Received 1 June 2015; accepted 27 June 2015; published online 21 July 2015)

\begin{abstract}
New ceramic $\mathrm{Ca}_{3} \mathrm{Co}_{3.9} \mathrm{O}_{9+\delta} / \mathrm{CaMn}_{0.97} \mathrm{~W}_{0.03} \mathrm{O}_{3-\delta}$ thermoelectric generators with different cross section areas $A_{p}$ and $A_{n}$ of the p- and the n-type leg are fabricated, characterized, and tested at high temperatures in long-term tests. The variation of the measured power output and the efficiency with changing $A_{p} / A_{n}$ ratio is discussed and compared with calculations based on the measured material properties. The highest conversion efficiencies are reached for ratios close to the one predicted by the compatibility approach, whereas an improper choice of $A_{p} / A_{n}$ leads to a strong reduction of the efficiency. A volume power density of $1.4 \mathrm{~W} / \mathrm{cm}^{3}$ and an efficiency of $1.08 \%$ are found for the most promising generator (temperature difference $\Delta T=734 \mathrm{~K}$ and $A_{p} / A_{n}=1.12$ ). The results reveal the major importance of the $A_{p} / A_{n}$ ratio for the conversion efficiency and subsequently cost and weight reduction issues, both crucial for a large scale application of thermoelectric converters. Additionally, the oxide generators proved to be very reliable, as after more than $110 \mathrm{~h}$ of high temperature energy conversion, no degradation is observable. C 2015 AIP Publishing LLC. [http://dx.doi.org/10.1063/1.4926476]
\end{abstract}

\section{INTRODUCTION}

Even modern industrial casting processes consume several GWh of electricity and release waste heat from large induction furnaces to melt tons of metals. For the implementation of thermoelectricity in such a potential high temperature energy harvesting application, the fabrication of reliable, economical, and efficient thermoelectric generators is necessary. Oxides are very promising candidates as the operation temperatures of other thermoelectric materials like bismuth telluride $\left(T_{\max } \leq 550 \mathrm{~K}\right){ }^{1}{ }^{1}$ skutterudites $\left(T_{\max }<673 \mathrm{~K}\right){ }^{2,3}$ and halfHeusler compounds $\left(T_{\max } \leq 850 \mathrm{~K}\right)^{4-6}$ are moderately low and the materials deteriorate in oxidizing atmospheres.

In literature, various high temperature oxide converters were manufactured and characterized, ${ }^{7-13}$ with many of them based on the promising p-type misfit-layered $\mathrm{Ca}_{3} \mathrm{Co}_{4} \mathrm{O}_{9}$ and its $\mathrm{Gd}$ or $\mathrm{Bi}$ substituted compounds. The cobalt oxide was combined with n-type materials like $\mathrm{La}_{0.9} \mathrm{Bi}_{0.1} \mathrm{NiO}_{3},{ }^{8,14,15}$ $(\mathrm{ZnO})_{7} \mathrm{In}_{2} \mathrm{O}_{3},{ }^{7} \mathrm{Zn}_{0.98} \mathrm{Al}_{0.02} \mathrm{O},{ }^{11}$ and $\mathrm{CaMnO}_{3}$ based materials with $\mathrm{La}, \mathrm{Nd}, \mathrm{Mo}, \mathrm{Sm}$, or Nb substitution. ${ }^{16-19}$ The highest measured power densities so far are $0.45 \mathrm{~W} / \mathrm{cm}^{2}$ or $0.96 \mathrm{~W} / \mathrm{cm}^{3}$ for a $\mathrm{Ca}_{2.7} \mathrm{Bi}_{0.3} \mathrm{Co}_{4} \mathrm{O}_{9} / \mathrm{LaNiO}_{3}$ generator for a hot side temperature of $1073 \mathrm{~K}^{15}$ Besides, for a $\mathrm{Ca}_{2.7} \mathrm{Bi}_{0.3} \mathrm{Co}_{4} \mathrm{O}_{9} / \mathrm{La}_{0.9} \mathrm{Bi}_{0.1} \mathrm{NiO}_{3}$ module, a volume and a mass power density of $0.66 \mathrm{~W} / \mathrm{cm}^{3}$ and $0.14 \mathrm{~W} / \mathrm{g}$ were measured. ${ }^{8}$ However, converter efficiencies are rarely reported and the necessary adjustment by applying the compatibility approach for the optimization of the generator

\footnotetext{
${ }^{\text {a) }}$ Author to whom correspondence should be addressed. Electronic mail: spopuloh@gmail.com
}

dimensions and geometries is still an open task. While for a $\mathrm{Ca}_{2.7} \mathrm{Bi}_{0.3} \mathrm{Co}_{4} \mathrm{O}_{9} / \mathrm{Ca}_{0.9} \mathrm{Yb}_{0.1} \mathrm{MnO}_{3}$ module, a theoretical conversion efficiency of about $3 \%$ was predicted from the material properties, ${ }^{20}$ much lower measured efficiencies of $0.15 \%-0.082 \%$, and $0.37 \%$ were stated for $\mathrm{La}_{1.98} \mathrm{Sr}_{0.02} \mathrm{CuO}_{4} / \mathrm{CaMn}_{0.98} \mathrm{Nb}_{0.02} \mathrm{O}_{3}{ }^{21,22}$ and $\mathrm{Ca}_{3} \mathrm{Co}_{4} \mathrm{O}_{9} /$ $2 \% \mathrm{Al}$-doped $\mathrm{ZnO}$ converters, ${ }^{23}$ respectively. For the latter converter, a much better efficiency of $1.16 \%$ could be achieved for additional segmentation of the p-type leg with $\mathrm{Ti}_{0.3} \mathrm{Zr}_{0.35} \mathrm{Hf}_{0.35} \mathrm{CoSb}_{0.8} \mathrm{Sn}_{0.2} \cdot{ }^{23}$ For a single $p$-type segmented leg constructed from $\mathrm{Ca}_{2.8} \mathrm{Lu}_{0.15} \mathrm{Ag}_{0.05} \mathrm{Co}_{4} \mathrm{O}_{9}$ and the halfHeusler alloy $\mathrm{Ti}_{0.3} \mathrm{Zr}_{0.35} \mathrm{Hf}_{0.35} \mathrm{CoSb}_{0.8} \mathrm{Sn}_{0.2}$, a maximum efficiency of about $5 \%$ was measured at $T_{h}=1153 \mathrm{~K}$. Although it is the highest reported value for an oxide based leg, the experimental value reaches only $65 \%$ of the theoretical efficiency due to parasitic resistances. ${ }^{24}$ A further increase of the total efficiency can also be achieved by cascading of different conventional converts with improved efficiency.

This work focuses on the enhancement of nonsegmented oxide converters by changing the $A_{p} / A_{n}$ ratio and shows that experimental converter efficiencies larger than $1 \%$ can be reached, which are comparable to state-of-the-art segmented oxide converters. Diverse high $T$ oxide converters are manufactured, but usually no attention is put on adjusting the $A_{p} / A_{n}$ ratio or determining the efficiency. Hence, in this work, we illustrate the importance of optimizing oxide converters according to the $A_{p} / A_{n}$ ratio and address the determination of the efficiency using the compatibility approach introduced in the following.

As stated by Snyder et al, the efficiency of a thermoelectric material depends on the reduced current density 
$u$ that is defined as the ratio of electrical current density $(J)$ to conductive heat flux. ${ }^{25}$ For a specific reduced current density $u$, the efficiency reaches a maximum. This ratio is called the compatibility factor $s$. It can be calculated from the materials properties and depends on temperature $(T)$. The reduced current density $u^{*}$, leading to the highest efficiency of the entire leg, has to be chosen considering $s(T)$. For the combination of p- and n-type material, it is important that both legs can work with their most efficient $u^{*}$. In this case, the legs are compatible with each other. This compatibility can be reached by adjusting the ratio $A_{p} / A_{n}$ of the cross section areas $A_{p}$ and $A_{n}$ of the p- and the n-type leg. However, the most efficient $A_{p} / A_{n}$ ratio depends on the hot side temperature $T_{h}$ and the cold side temperature $T_{c}$ of the legs, which can strongly fluctuate in time for, e.g., mobile applications. ${ }^{26}$ Therefore, knowledge about the dependency of the efficiency on the cross section ratio $A_{p} / A_{n}$ and its temperature dependency can help to tailor thermoelectric converters accordingly. On top of that, an adjustment allows for cost, volume, or weight reduction, making the converters more economical.

In this work, the effect of a varied cross section area ratio $A_{p} / A_{n}$ on the efficiency of thermoelectric generators is investigated theoretically and experimentally. Therefore, a series of 4-leg thermoelectric generators with $A_{p} / A_{n}=0.74$, $1.01,1.12,1.24$, and 2.74 is fabricated from the p-type $\mathrm{Ca}_{3} \mathrm{Co}_{3.9} \mathrm{O}_{9.3+\delta}$ and the n-type $\mathrm{CaMn}_{0.97} \mathrm{~W}_{0.03} \mathrm{O}_{3-\delta}$. These oxides stand out due to their relatively high thermoelectric Figure of Merits $(Z T)$, non-toxicity, and high chemical and temperature stability $\left(T_{\max } \approx 1200 \mathrm{~K}\right)^{27}$ in air. Moreover, their low material and synthesis costs ${ }^{28,29}$ allow the fabrication of competitive thermoelectric converters. As mechanically stable generators with low contact resistances can be fabricated using Ag-paste mixed with cobalt oxide powder soldered via hot pressing, ${ }^{15}$ this material combination provides a good basis for studying the influence of the leg geometry. For both materials, the Seebeck coefficient $(\alpha)$, the electrical $(\sigma)$, and the thermal conductivities $(\kappa)$ are measured and used to calculate the most efficient cross section ratio, the maximum power output, and the efficiency for different temperature gradients. Furthermore, a comparison of the theoretical key figures and the experimental data is made.

\section{THEORETICAL MODEL}

According to Snyder et al., ${ }^{25,30}$ the conversion efficiency $\eta(T)=\eta_{r} \eta_{C}$ of a material at temperature $T$ can be calculated from the Carnot efficiency $\eta_{C}=\frac{T_{h}-T_{c}}{T_{h}}$ and the reduced efficiency

$$
\eta_{r}=\frac{u(\alpha-u \kappa / \sigma)}{u \alpha+1 / T}
$$

using the average values $\sigma, \alpha, \kappa$, and $T$ of the considered interval $\Delta T=T_{h}-T_{c}$. The reduced efficiency depends on the relative current density $u=\frac{J}{\kappa \nabla T}$. It reaches a maximum value $\eta_{r}^{\max }$ for $u$ equal to the compatibility factor $s=\frac{\sqrt{1+Z T}-1}{\alpha T}$, where $Z=\frac{\sigma \alpha^{2}}{\kappa}$. To reach a high total leg efficiency, $u$ should be close to $s$ in the whole leg.
Within the leg, $u(\mathrm{~T})$ is fixed by the solution of the onedimensional steady-state heat equation, which can be approximated by a recursive formula. ${ }^{30}$ For the recursive calculation, the starting value $u_{0, h}$ at the hot side of the leg can be chosen freely, which corresponds to choosing the external load resistance in the experimental setup. Based on the chosen $u(T)$, the total leg efficiency $\eta_{\text {leg }}=\int_{T_{c}}^{T_{h}} \eta(T) d T$ is found by integration over the leg's temperature range. Finally, the most efficient relative current $u^{*}(T)$, which maximizes the efficiency $\eta_{l e g}$ of the whole leg can be found via a variation of $u_{0, h}$. More details and model calculations can be found elsewhere. ${ }^{30-32}$

In a thermoelectric converter, the same electrical current $I=J_{p} A_{p}=-J_{n} A_{n}$ flows in both legs, and thus,

$$
\frac{A_{p}}{A_{n}}=\frac{-J_{n}}{J_{p}}=\frac{-\int_{T_{c}}^{T_{h}} u_{n} \kappa_{n} d T}{\int_{T_{c}}^{T_{h}} u_{p} \kappa_{p} d T} .
$$

From this equation, the ratio $\left(A_{p} / A_{n}\right)^{*}$ for which both legs work with their highest efficiency can be calculated using $u_{p}=u_{p}^{*}$ and $u_{n}=u_{n}^{*}$. Additionally, for a generator with arbitrary $A_{p} / A_{n}$ the equation fixes $u_{n}$ as soon as $u_{p}$ is chosen. These $u$ determine the efficiencies $\eta_{p}$ and $\eta_{n}$ of the single legs, and thus, the power output $P=\eta_{p} Q_{p}^{h}+\eta_{n} Q_{n}^{h}$ and the efficiency

$$
\eta_{n+p}=\frac{\eta_{p} Q_{p}^{h}+\eta_{n} Q_{n}^{h}}{Q_{p}^{h}+Q_{n}^{h}}
$$

of the whole generator. Thereby, the heat flow

$$
Q=J \Phi A
$$

is determined by the thermoelectric potential $\Phi=\alpha T+\frac{1}{u}$ and $Q_{p}^{h}$ and $Q_{n}^{h}$ are the heat flows entering the hot sides of the legs. Finally, the maximum of the power output or the efficiency is found by a variation of $u_{p}$. The associated reduced current densities are $u_{p}^{* *}$ and $u_{n}^{* *}$.

While for the compatibility approach, $u(T)$ is considered to depend only on $T$, a related concept developed in the $1960 \mathrm{~s}^{33}$ shows that $u$ is also a function of the local electrochemical potential. Thus, the latter approach is more accurate, but our results show that Snyder's approximation of $u(T)$ forms a good basis for the $A_{p} / A_{n}$ optimization.

\section{MATERIALS AND EXPERIMENTAL METHODS}

To synthesize $0.14 \mathrm{~mol} \mathrm{Ca}_{3} \mathrm{Co}_{3.9} \mathrm{O}_{9.3+\delta}, 0.549 \mathrm{~mol} \mathrm{Co}$ metal and $0.42 \mathrm{~mol} \mathrm{CaCO}_{3}$ were suspended in about $300 \mathrm{ml}$ water. Under cooling, $400 \mathrm{ml}$ of nitric acid ( $>69 \%$ aq.) were added continuously. The resulting clear pink solution was transferred into about $200 \mathrm{ml}$ of an aqueous solution of $1.94 \mathrm{~mol}$ citric acid. After drying at $573 \mathrm{~K}$, the residuum of the gel was crushed and calcined in air at $1073 \mathrm{~K}$ for $10 \mathrm{~h}$. Next, a pellet $(\varnothing=20 \mathrm{~mm} \times \mathrm{h}=11 \mathrm{~mm})$ was sintered in vacuum by Spark Plasma Sintering (FCT-HP D 10, $50 \mathrm{MPa}$, 
heating $100 \mathrm{~K} / \mathrm{min}$ to $1123 \mathrm{~K}$, then $25 \mathrm{~K} / \mathrm{min}$ to $1153 \mathrm{~K}$ and $5 \mathrm{~min}$ holding time. Cooling at $25 \mathrm{MPa}$ and $25 \mathrm{~K} / \mathrm{min}$ to $773 \mathrm{~K}$ followed by free cooling). Finally, the legs for the generators and samples for the material characterizations were cut from the same pellet. The samples were postannealed in air at $1123 \mathrm{~K}$ for $20 \mathrm{~h}$ to ensure similar and homogeneous oxygen content. The highest $Z T$ was found perpendicular to the pressing direction. Therefore, the modules were manufactured accordingly $(Q \perp$ pressing direction). The material for the n-type legs $\left(\mathrm{CaMn}_{0.97} \mathrm{~W}_{0.03} \mathrm{O}_{3-\delta}\right)$ of the generators was synthesized via a citrate process. ${ }^{29} \mathrm{~A}$ hydrostatically pressed $(2000$ bar $)$ green body $(\varnothing=40 \mathrm{~mm}$ $\times \mathrm{h}=9 \mathrm{~mm}$ ) was produced and pre-sintered at $1373 \mathrm{~K}$ in a muffle furnace. Afterwards, the samples for the characterization and the legs for the modules were cut from the pellet and sintered in air with a maximum temperature of $1573 \mathrm{~K}$ and a dwelling time of $5 \mathrm{~h}$.

Both materials were characterized in air from room temperature to $1163 \mathrm{~K}$. The Seebeck coefficient $(\alpha)$ and the electrical conductivity $(\sigma)$ were measured using the Ozawa Science RZ2001i system. The thermal conductivity $\left(\kappa=\rho \lambda C_{p}\right)$ was determined using Laser Flash Absorption (LFA; Netzsch LFA 457 Microfash) and a differential scanning calorimeter (DSC; Netzsch DSC 404C) for the measurement of the thermal diffusivity $(\lambda)$ and the specific heat $\left(C_{p}\right)$, respectively. For the calculation of $\kappa$, the endothermic $C_{p}$-peak of the p-type material was excluded. The densities of the samples were determined by the Archimedes method.

A series of four-leg half-skeleton modules (inset of Figure 3) was fabricated from $\mathrm{Ca}_{3} \mathrm{Co}_{3.9} \mathrm{O}_{9.3+\delta}$ and $\mathrm{CaMn}_{0.97} \mathrm{~W}_{0.03} \mathrm{O}_{3-\delta}$. For each module, the total leg crosssectional area of the four legs was chosen to be $40.77(6) \mathrm{mm}^{2}$ with varying cross section ratio $\left(A_{p} / A_{n}=0.74,1.01,1.12\right.$, 1.24 , and 2.74). The legs were electrically connected by Agsheets and joined with a Ag-paste containing $6 \mathrm{wt} \%$ of $\mathrm{Ca}_{2.97} \mathrm{Bi}_{0.03} \mathrm{Co}_{4} \mathrm{O}_{9+\delta}$ power. ${ }^{8,19}$ The whole module was soldered in air at $1123 \mathrm{~K}$ and $6.3 \mathrm{MPa}$ uniaxial pressure.

All generators were characterized in a thermoelectric test stand under identical conditions. The hot-side temperature $T_{h}$ was measured with two thermocouples fixed on the inner side of the $\mathrm{Al}_{2} \mathrm{O}_{3}$ plate close to the p- and the n-type legs, while the cold side thermocouples were placed between the silver contact of the p-/n-type leg and the cooling plate. Current-voltage characteristics were recorded at different temperature differences using a Keithley 2440 SourceMeter (4-wire mode). Independent of the $A_{p} / A_{n}$ ratio all modules should supply the same open circuit voltage $V_{O C}=\int_{T_{c}}^{T_{h}} \alpha(T) d T$ for the same $T_{h}$ and $T_{c}$. To compensate for small temperature drifts, the power output was extracted from an extrapolation of the measured $P-V_{O C}$-dependency to the same $V_{O C}$ for all converters (cf. Section I in supplementary material ${ }^{34}$ ). In Table I, $V_{O C}$ and the corresponding $T_{h}$ and $T_{c}$ measured for the module with $A_{p} / A_{n}=1.12$ are presented for different heater temperatures. Finally, the module efficiencies were calculated using the theoretical total heat flow entering the legs at the hot side. Here, the flow was calculated from the materials' properties, the leg dimensions, and the measured $T_{c}$ and $T_{h}$ via Eq. (4), using $u$ or $\Phi$ optimized for maximum power output.
TABLE I. Temperature of hot $T_{h}$ and cold side $T_{c}$ with corresponding $V_{O C}$ measured for the module with $A_{p} / A_{n}=1.12$.

\begin{tabular}{lcccccccc}
\hline \hline$T_{h}(\mathrm{~K})$ & 369 & 464 & 563 & 658 & 759 & 856 & 949 & 1051 \\
\hline$T_{c}(\mathrm{~K})$ & 297 & 300 & 303 & 308 & 310 & 315 & 321 & 324 \\
$V_{O C}(\mathrm{~V})$ & 0.030 & 0.071 & 0.115 & 0.161 & 0.210 & 0.259 & 0.310 & 0.364 \\
\hline \hline
\end{tabular}

The contact resistivity $\rho_{c}$ of the oxide-Ag composite (6 wt. \% $\mathrm{Ca}_{2.97} \mathrm{Bi}_{0.03} \mathrm{Co}_{4} \mathrm{O}_{9+\delta}$ ) interface was determined on series of 7 legs with equal cross section but variable length. Ag contacts were joined to the legs with the same soldering procedure used for the modules. Additionally, a series of $\mathrm{Ca}_{2.97} \mathrm{Bi}_{0.03} \mathrm{Co}_{4} \mathrm{O}_{9+\delta}$ legs was soldered without pressure $(5 \mathrm{~h}$ at $1123 \mathrm{~K}$ ). The length $(l)$ of the legs was determined prior to soldering. The leg resistances $R_{\text {tot }}$ were measured in 4-probe geometry with both clamps connected to the supply leads. As $R_{\text {tot }}(l)=R_{\text {leg }}(l)+2 R_{\text {contact }}$ changes linear with 1 the contact resistance $R_{c}$, and thus, the resistivity $\rho_{c}=R_{c} A$ can be extracted from a fit.

\section{RESULTS AND DISCUSSION}

The materials' properties of both oxides were investigated to understand the dependency of the generator efficiency on the $A_{p} / A_{n}$ ratio via theoretical calculations and to fabricate generators with reasonable $A_{p} / A_{n}$ ratios. The misfit-layered cobaltite $\mathrm{Ca}_{3} \mathrm{Co}_{3.9} \mathrm{O}_{9.3+\delta}$ and the perovskitetype manganite $\mathrm{CaMn}_{0.97} \mathrm{~W}_{0.03} \mathrm{O}_{3-\delta}$ exhibit $99 \%$ (4.63(10) $\mathrm{g} / \mathrm{cm}^{3}$ ) and $97 \%\left(4.52(18) \mathrm{g} / \mathrm{cm}^{3}\right)$ of the theoretical densities, ${ }^{35}$ respectively. The electrical conductivity, the absolute Seebeck coefficient, and the thermal conductivity of the two oxides are of comparable magnitude (Figures 1(a)-1(c)). For both materials, the experimental data are in agreement with literature, ${ }^{28,29}$ although for the cobaltite $\kappa$ is in the upper region of the literature values as the measurement direction is across the aligned particles. ${ }^{36}$ Because of the higher $\sigma$ at elevated temperatures, the Figure of merit $Z T=$ $\frac{\alpha^{2} \sigma}{\kappa} T$ of the p-type material is superior to that of the n-type material (Figure 1(d)). The $\mathrm{CaMn}_{0.97} \mathrm{~W}_{0.03} \mathrm{O}_{3-\delta}$ samples reached a peak value of $Z T=0.11$ at $1123 \mathrm{~K}$, which is comparable with other substituted manganites. ${ }^{28}$ At the same temperature, the $\mathrm{Ca}_{3} \mathrm{Co}_{3.9} \mathrm{O}_{9.3+\delta}$ sample reached a $Z T=$ 0.17 , which is in agreement with the smaller values reported in literature. ${ }^{28}$

Additionally, in Figure 1(e), the compatibility factors $s(T)$ of the oxides are presented, with $s_{p}$ showing the stronger temperature dependence. In comparison, the most efficient reduced current densities $u_{p}^{*}(T)$ and $u_{n}^{*}(T)$ calculated for $\Delta T=1051 \mathrm{~K}-324 \mathrm{~K}$ show a relatively flat temperature rise. Because $J$ is constant within the legs, and the conductive heat flows change moderately, the slope of both $u$ is rather small.

To allow both legs to work with the most efficient reduced current densities $u_{p}^{*}$ and $u_{n}^{*}$, the ratio of the cross section areas should be equal to $\left(A_{p} / A_{n}\right)^{*}$ (see Eq. (2)). In Figures 2(a) and 2(b), this cross section ratio and the corresponding p-n-couple efficiency $\eta_{p+n}^{*}$ are presented for different $T_{h}$ and $T_{c}$. Here, $\eta_{p+n}^{*}$ illustrates the potential of this 


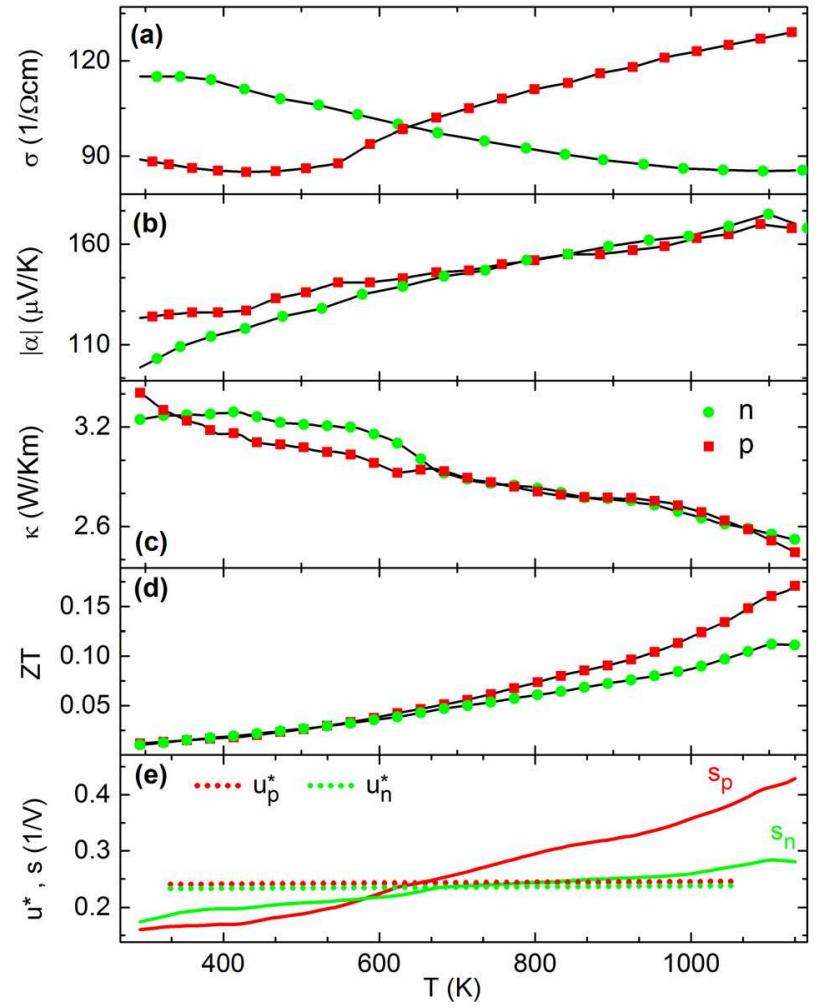

FIG. 1. Thermoelectric properties of $\mathrm{Ca}_{3} \mathrm{Co}_{3.9} \mathrm{O}_{9.3+\delta}$ (p-type) and $\mathrm{CaMn}_{0.97} \mathrm{~W}_{0.03} \mathrm{O}_{3}$ (n-type): (a) electrical conductivity $\sigma$, (b) absolute Seebeck coefficient $|\alpha|$, (c) thermal conductivity $\kappa$, (d) Figure of Merit $Z T$, and (e) compatibility factor $s$ with most efficient reduced current densities for $\Delta T=1051 \mathrm{~K}-324 \mathrm{~K}$. Based on the uncertainties in the distance measurement between the electrode-contact wires and the sample dimensions as well as non-optimal thermal contacts, uncertainties of $\Delta \sigma=7 \%$ and $\Delta \alpha=5 \%$ are expected. ${ }^{37}$ Error propagation of the uncertainties of the density, the thermal diffusivity $(\Delta \lambda=2 \%)$, and the heat capacity $\left(\Delta C_{p}<5 \%\right)$ results in uncertainties of $\Delta \kappa=5.5 \%$ and $\Delta Z T=13.4 \%$ (error bars are omitted for the sake of clarity).

material composition for different $\Delta T$, giving a maximum value of $1.48 \%$ at $\Delta T=1148-298 \mathrm{~K}$. The ratio $\left(A_{p} / A_{n}\right)^{*}$ tends to decrease with increasing $T_{h}$ and $T_{c}$ (Figure 2(a)). For a thermoelectric generator, the $A_{p} / A_{n}$ ratio is fixed and can only be adjusted to a single $\Delta T$. A series of generators was fabricated to investigate the influence of a non-adjusted $A_{p} / A_{n}$ ratio on the module performance. Their ratios are presented in Figure 2(c) in comparison with the $\left(A_{p} / A_{n}\right)^{*}$-ratios calculated for the experimentally applied $\Delta T$. The ratios 1.12 and 1.24 are equal or close to $\left(A_{p} / A_{n}\right)^{*}$ between 370 and $660 \mathrm{~K}$, whereas the ratio 1.01 is adjusted to $T_{h}$ around $920 \mathrm{~K}$.

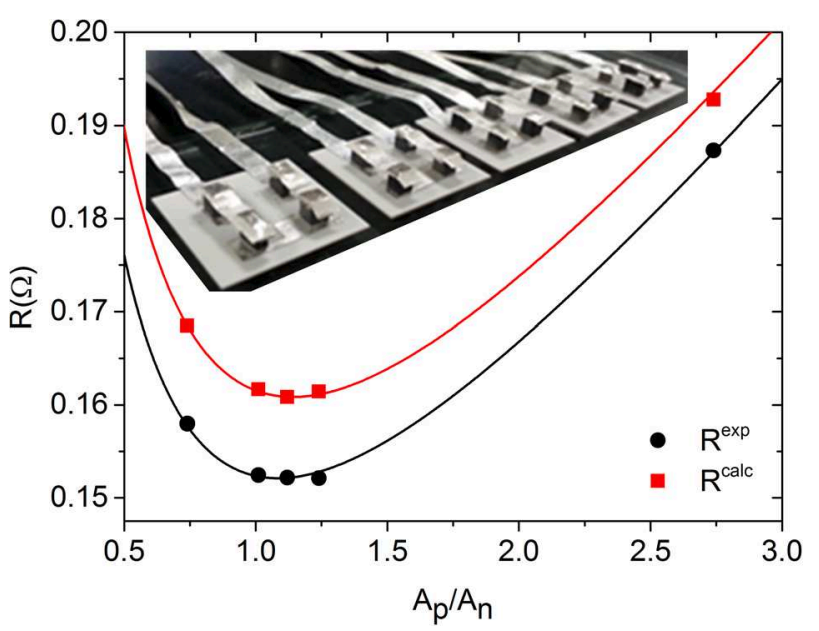

FIG. 3. Room temperature module resistances $R^{\exp }$ measured for different $A_{p} / A_{n}$ ratios (black circles) with corresponding fit of $R_{a v}^{\text {calc }}\left(A_{p} / A_{n}\right)$ (Eq. (5)). Additionally, the module resistances $R^{\text {calc }}$ calculated from the leg dimensions and resistivities are presented (squares). The red line shows the resistance $R_{a l}^{\text {calc }}\left(A_{p} / A_{n}\right)$ calculated for the average module area and leg height. For $R^{\text {calc }}$, an additional contribution from the contact resistance smaller than $4 \mathrm{~m} \Omega$ has to be considered. In the inset, a picture of the fabricated 4-leg generators is presented.

In contrast, the ratios 0.74 and 2.74 are smaller or much larger than $\left(A_{p} / A_{n}\right)^{*}$, respectively.

A picture of the fabricated modules and the measured room temperature resistances $R^{\exp }$ are presented in Figure 3. A minimal value of $152.12 \mathrm{~m} \Omega$ was found for $A_{p} / A_{n}=$ 1.24. This is about $7 \%$ larger than the room temperature resistance $R^{\text {calc }}$ calculated from the geometry of the four generator legs connected in series. As a guide for the eye, the continuous function

$$
\begin{aligned}
R_{a v}^{\text {calc }}\left(\frac{A_{p}}{A_{n}}\right) & =2\left(R_{n}+R_{p}\right) \\
& =2 \frac{d}{A_{p}+A_{n}}\left(\frac{1}{\sigma_{n}}\left(1+\frac{A_{p}}{A_{n}}\right)+\frac{1}{\sigma_{p}}\left(\frac{1}{\frac{A_{p}}{A_{n}}}+1\right)\right)
\end{aligned}
$$

is plotted, which describes the summarized room temperature resistances for the $\mathrm{p}$ - and the n-type legs $R_{p}$ and $R_{n}$ with varying $A_{p} / A_{n}$ using the constant average leg height $d=0.411(4) \mathrm{cm}$ and the constant average $A_{p}+A_{n}$ of all modules (Figure 3 ). The very small discrepancy between the average function $R_{a v}^{\text {calc }}$ and $R^{\text {calc }}$ emphasizes the
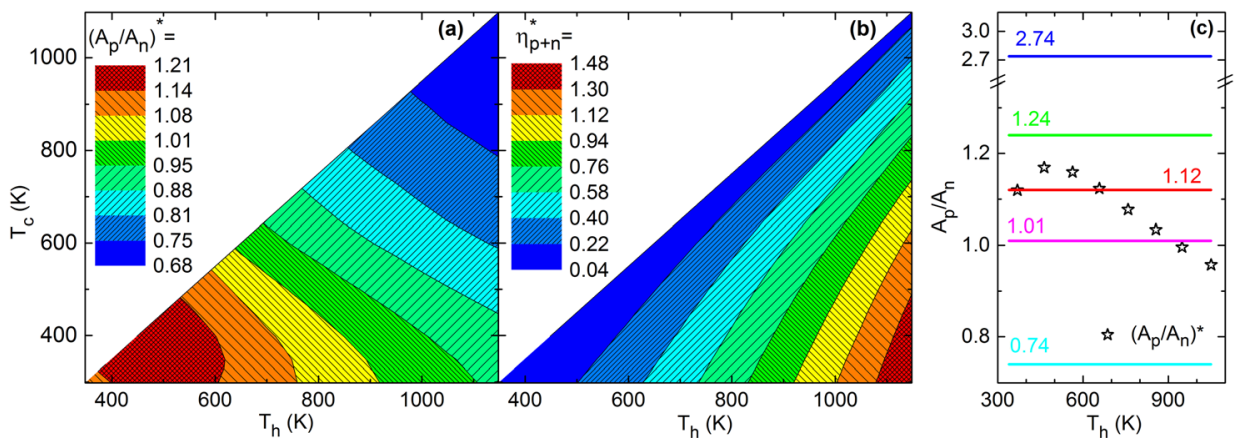

FIG. 2. Cross section area $\left(A_{p} / A_{n}\right)^{*}$ ensuring the highest efficiencies of both legs (a) and maximum efficiency $\eta_{p+n}^{*}$ of a generator with ratio $\left(A_{p} / A_{n}\right)^{*}$ for different cold $T_{c}$ and hot side temperatures $T_{h}$ (b). Additionally, $\left(A_{p} / A_{n}\right)^{*}$ for the used experimental $T_{h}$ and $T_{c}$ are shown in comparison with the ratios of the fabricated generators (lines) (c). 
precise cutting of the leg geometry and the homogeneity of the material.

Additionally, the contact resistances of the oxide-Ag interfaces are expected to contribute to $R^{\text {calc }}$. For $\mathrm{CaMn}_{0.97} \mathrm{~W}_{0.03} \mathrm{O}_{3-\delta}$, a contact resistivity of $\rho_{n}^{c}=0.04(2) \mathrm{m} \Omega$ $\mathrm{cm}^{2}$ was found for a series of legs soldered in the same way as the modules. As the contact resistivity of the Ag$\mathrm{Ca}_{3} \mathrm{Co}_{3.9} \mathrm{O}_{9.3+\delta}$ contacts soldered this way is too small for a reliable determination, it was determined for a $\mathrm{Ag}-\mathrm{Ca}_{2.97} \mathrm{Bi}_{0.03} \mathrm{Co}_{4} \mathrm{O}_{9+\delta}$-interface soldered without pressure. Here, a contact resistivity of $\rho_{p}^{c}=0.025(16) \mathrm{m} \Omega \mathrm{cm}^{2}$ was found. Adding the contacts resistances leads to contact resistance of about $R_{c}=4 \mathrm{~m} \Omega$ per generator. Because this value amounts to only about $2 \%$ of the total module resistance, its omission in the theoretical calculations is reasonable. The observed difference between $R^{\text {calc }}$ and $R^{\exp }$ does not result from the contacts, but a change of the resistance of the p-type material during the hot pressing of the module is observed (cf. Section II in supplementary material $^{34}$ ).

The measured power outputs of the fabricated generators is presented in Figures 4(a) and 4(b) to analyze the effect of the $A_{p} / A_{n}$ ratio. While only the absolute power outputs $P_{1.01}$ and $P_{2.74}$ of the generators with $A_{p} / A_{n}=1.01$ and 2.74 are depicted in Figure 4(a), small differences between all the generators are displayed in Fig. 4(b) via a normalization to $P_{1.01}$. Depending on the $A_{p} / A_{n}$ ratio, different temperature dependencies are observed, although the power outputs of all generators increase with $T_{h}$ (or $\Delta T$ ). For $T_{h}$ around $400 \mathrm{~K}$, the highest power outputs $(1.45 \mathrm{~mW})$ are produced by generators with the ratios 1.12 and 1.24 , whereas the ratios 1.12 and 1.01 are most suitable for $T_{h}$ around $1051 \mathrm{~K}\left(P_{\max }=\right.$

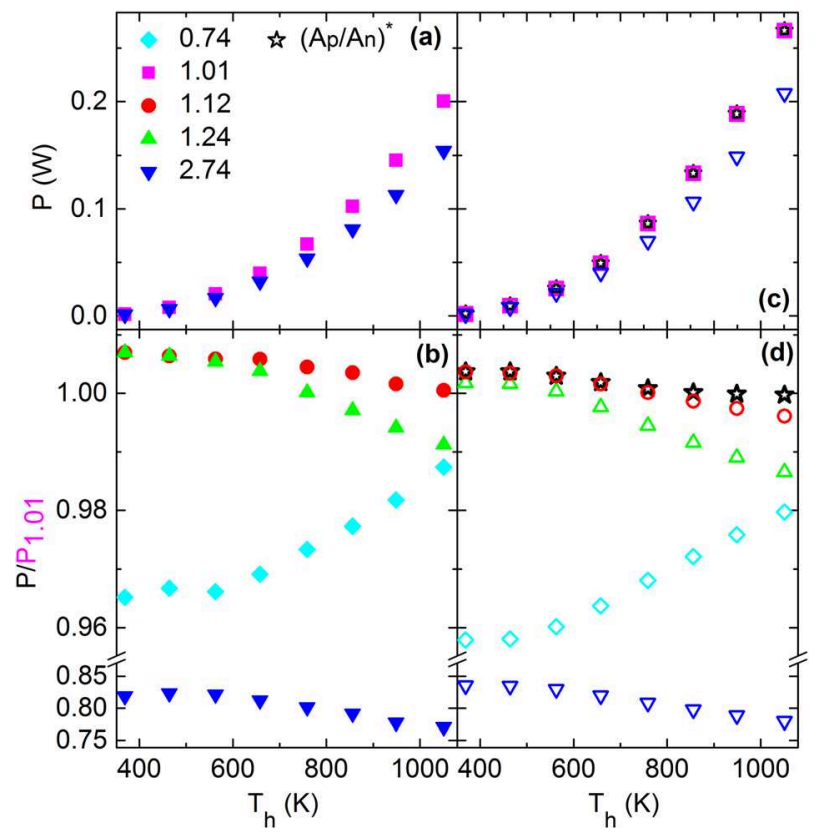

FIG. 4. Temperature dependent experimental power outputs for $A_{p} / A_{n}=1.01$ and 2.74 (a). A normalization of the experimental power outputs to the power output measured for the generator with $A_{p} / A_{n}=1.01$ reveals also small differences between the different ratios (b). In comparison to the experimental values (open symbols), the corresponding theoretical values (filled symbols) are presented in (c) and (d). Additionally, the values of a generator with $\left(A_{p} / A_{n}\right)^{*}$ adapted to each temperature difference are shown.
$200.4 \mathrm{~mW}$ ). In contrast, a strong reduction of the power output is observed for the ratios 0.74 and 2.74, which differ strongly from $\left(A_{p} / A_{n}\right)^{*}$. At the largest $\Delta T$, a reduction of up to $23 \%$ is found for $P_{2.74}$ compared to $P_{1.01}$.

To better understand the observed trends, the theoretical power outputs are calculated based on the measured $T_{h}$ and $T_{c}$ as well as the materials properties (Figures 4(c) and 4(d)). Additionally, for every $T_{h}$, the theoretical power output of a module with the ratio $\left(A_{p} / A_{n}\right)^{*}$ is depicted. The theoretical power output exceeds the experimental ones by up to $35 \%$. However, the model describes the relative changes very well (compare Figures 4(b) and 4(d)). In agreement with the experimental values, it predicts the strongly reduced power outputs for the ratios 0.74 and 2.74. Furthermore, it also confirms that the highest power outputs are produced for the ratios 1.12 and 1.01 in the low- and the high-temperature regions, respectively. Comparing the power outputs with the one of $\left(A_{p} / A_{n}\right)^{*}$, it can be concluded that the closer $A_{p} / A_{n}$ is to $\left(A_{p} / A_{n}\right)^{*}$ the higher is the power output (cf. Figure 2(c)).

This dependency of the power output on the $A_{p} / A_{n}$ ratio can be understood by having a closer look at the reduced current densities within the legs. In line with the introduced theoretical model, we will use the symbols $u_{p}^{*}$ and $u_{n}^{*}$ for the reduced current densities, leading to the highest leg efficiencies and the symbols $u_{p}^{* *}$ and $u_{n}^{* *}$ for the reduced current densities that lead to the highest efficiency of the entire generator. The total efficiency of one leg is calculated from the reduced efficiency $\eta_{r}(u)$, which reaches to a maximum value $\eta_{r, \max }$ for $u=s$. While the slope of the compatibility factor $s(T)$ is fixed by the material properties of the two oxides, the slope of $u(T)$ is determined by the heat equation, cf. Figure 5(a). The highest leg efficiency is reached for the $u^{*}$, which is closest to $s$ within the whole temperature range. For a generator with $\left(A_{p} / A_{n}\right)^{*}$, this is the case for both legs, but for different ratios, $u_{p}^{* *}$ and $u_{n}^{* *}$ differ from $u_{p}^{*}$ and $u_{n}^{*}$. This is illustrated in Figures 5(a) and 5(b). For a generator with ratio $A_{p} / A_{n}=1.01$, the $\eta_{r}$ and $u^{* *}$ nearly coincide with

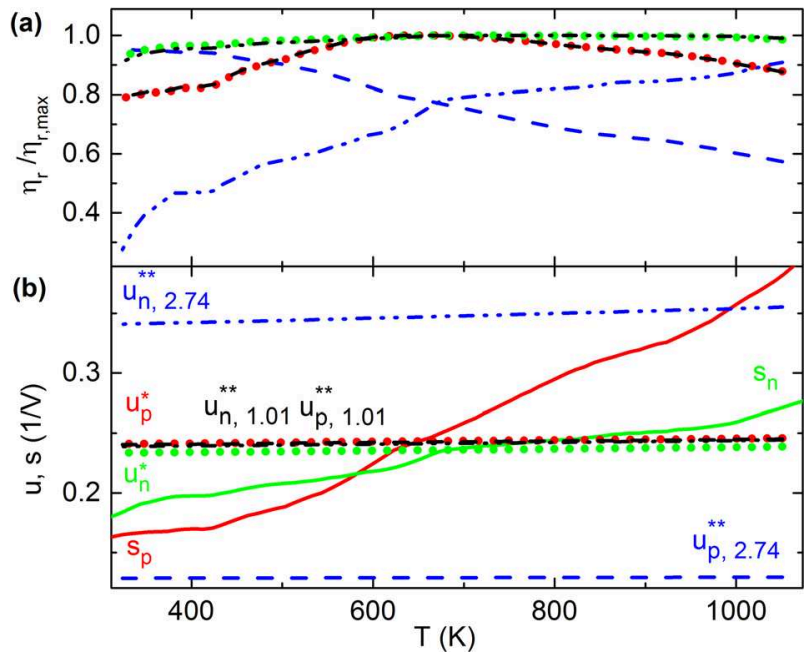

FIG. 5. (a) Local reduced efficiency $\eta_{r}$ normalized to the maximum reduced efficiency $\eta_{r, \max }$ of the material with (b) corresponding reduced current densities $u^{*}$ and compatibility factors $s$ calculated for $\Delta T=1133 \mathrm{~K}-293 \mathrm{~K}$. 
the values of a generator with $\left(A_{p} / A_{n}\right)^{*}=0.96$. In contrast, for $A_{p} / A_{n}=2.74$, the $u_{p}^{* *}$ and $u_{n}^{* *}$ deviate strongly from $u_{p}^{*}$ and $u_{n}^{*}$ and $\eta_{r} / \eta_{r, \max }$ is reduced by up to $43 \%$ and $73 \%$ for the $\mathrm{p}$ - and the $\mathrm{n}$-leg, respectively. A closer look reveals that for the p-type leg the highest efficiency losses are observed at high T, where the ratio $u_{p}^{* *}$ between electrical current density and heat flux is smaller than $s_{p}$. Accordingly, the thermoelectric segment produces a Seebeck voltage, but the electrical current is much smaller than for $u=s$. Thus, the efficiency and the power output, which increase linearly with the current, are small. For the n-type leg, the efficiency is also reduced, but in this case, $u_{n}^{* *}$ is larger than $s_{n}$. Here, the decrease results from Joule heating that is proportional to the square of the current and thus more dominant for higher values. Consequently, the two legs cannot work efficiently at the same time, because for a given $u_{n}^{* *}$, the reduced current $u_{p}^{* *}$ is fixed by the $A_{p} / A_{n}$ ratio and thus in at least one leg the reduced current is unfavorable.

For the largest temperature difference $\Delta T=727 \mathrm{~K}$, the highest experimental power output is reached for the module with the ratio $A_{p} / A_{n}=1.12$ (cf. Figure 4). The measured maximum power output, the maximum power density normalized to the total cross-sectional area of the legs, the leg volume, and the mass of the thermoelectric legs are found to be $P_{\max }=200.4 \mathrm{~mW}, 0.49 \mathrm{~W} / \mathrm{cm}^{2}, 1.2 \mathrm{~W} / \mathrm{cm}^{3}$, and $0.26 \mathrm{~W} / \mathrm{g}$ for $\Delta T=727 \mathrm{~K}$ and $A_{p} / A_{n}=1.12$, respectively. These values are comparable with values found for other oxide converters. ${ }^{7,9,10}$ The measured volume and area power densities are even $24 \%$ and $9 \%$ larger than the most promising literature values of $0.96 \mathrm{~W} / \mathrm{cm}^{3}$ and $0.45 \mathrm{~W} / \mathrm{cm}^{2}$ found for a $\mathrm{Ca}_{2.7} \mathrm{Bi}_{0.3} \mathrm{Co}_{4} \mathrm{O}_{9} / \mathrm{LaNiO}_{3}$ generator with similar $T_{h}$ of $1073 \mathrm{~K}^{15}$

The power densities are not the most adequate parameters for a comparison of generator performance since they depend on the height of the legs $\left(l_{t o t}\right)$. Informative is the calculation of the Figure of Merit $Z_{M}=\frac{4 P_{\max }}{\mathrm{K} \Delta T^{2}}$ of the module, ${ }^{38}$ where $\mathrm{K}$ is the thermal conductance $\mathrm{K}=\frac{N}{l_{t o t}}\left(\overline{\kappa_{n}} A_{n}+\overline{\kappa_{p}} A_{p}\right)$, $N$ the number of p-n couples, and $\overline{\kappa_{n}}$ and $\overline{\kappa_{p}}$ the average values of the conductivities at the hot and the cold side of the legs. For the $A_{p} / A_{n}=1.12$ module at the highest applied temperature, a value of $Z_{M}=52 / \mu \mathrm{K}$ was calculated.

The efficiency calculation allows the direct estimation of the power output for a given heat flow, which is an important value for the application. The heat flow is calculated from the measured $T_{h}=1051 \mathrm{~K}$ and $T_{c}=324 \mathrm{~K}$, the leg dimensions and the materials properties via Eq. (4). However, $T_{h}$ seems to overestimate the actual temperature, because it was measured on the $\mathrm{Al}_{2} \mathrm{O}_{3}$-plate next to the legs. The measured open circuit voltage $\left(V_{O C}=\int_{T_{c}}^{T_{h}} \alpha(T) d T\right)$ leads to a value of $T_{h}^{V_{O C}}=970 \mathrm{~K}$, which is about $8 \%$ smaller than $T_{h}$. This difference might originate from the active cooling of the legs, which has only a small effect on $T_{h}$. For the generator with $A_{p} / A_{n}=1.12$ for $T_{h}$, a total heat flow of $Q_{t o t}=22.0 \mathrm{~W}$ and an experimental converter efficiency of $\eta_{p+n}^{T_{h}}=0.91 \%$ are found. In contrast, for $T_{h}^{V o c}$, a heat flow of $Q_{t o t}=19.7 \mathrm{~W}$ is calculated, and the measured efficiency reaches an even higher value of $\eta_{p+n}^{T_{h}^{V O C}}=1.02 \%$.
For the same $T_{h}$ and $T_{h}^{V_{O C C}}$, a detailed overview on the change of the experimental and the theoretical couple efficiency with the $A_{p} / A_{n}$ ratio is given in Figure 6. As discussed for the power output, also the generator efficiency decreases dramatically (up to 23\%) for $A_{p} / A_{n}$ deviating strongly from $\left(A_{p} / A_{n}\right)^{*}$. Experimental and theoretical values show a similar $A_{p} / A_{n}$ dependency. For $T_{h}$, the experimental efficiencies stay up to $25 \%$ below the theoretical values. In contrast, if the heat flow and the theoretical power output are calculated for $T_{h}^{V_{O C}}$, the difference between the theoretical and the experimental efficiencies is smaller than $3 \%$. Thus, $T_{h}^{V o c}$ seems to be the more reliable temperature.

From the theoretical calculations, it can be seen that $\left(A_{p} / A_{n}\right)^{*}$ does not lead to the maximum efficiency of the whole module, but it is obviously very close to it (Figure 6): compared to the optimal ratio for $T_{h}$, a $0.04 \%$ higher efficiency is found for an increase of the cross section area by $6 \%$. This can be explained by the definition of the generator efficiency (Eq. (3)). A change of the $A_{p} / A_{n}$ ratio changes the total heat flow and more important the weighting of the pand the n-leg efficiency that determines the power output $P=\eta_{p} Q_{p}^{h}+\eta_{n} Q_{n}^{h}$. For $A_{p} / A_{n}>\left(A_{p} / A_{n}\right)^{*}$, the larger $A_{p}$ increases the heat flow $Q_{p}=J_{p} \Phi_{p} A_{p}$ through the p-leg with the higher efficiency $\left(\eta_{p}\right)$, whereas $Q_{n}$ decreases with $A_{n}$. Additionally, the leg efficiencies of one or both legs decrease because $u_{n} \neq u_{n}^{*}$ or $u_{p} \neq u_{p}^{*}$. In case that the change of the heat flows dominates the change of leg efficiency, this can lead to a higher power output. Accordingly, higher values can be reached through $A_{p} / A_{n}$ ratio different from $\left(A_{p} / A_{n}\right)^{*}$, although the efficiencies of the single legs are smaller. Regarding the efficiency, it has to be considered that an increase of $A_{p} / A_{n}$ leads to a higher heat flow $Q=Q_{p}+Q_{n}$ for the same $\Delta T$, due to different $\Phi$ of the legs. Thus, it is possible that maximum efficiency and maximum power can be found at slightly different ratios. However, this difference is considered to be rather small. ${ }^{39}$

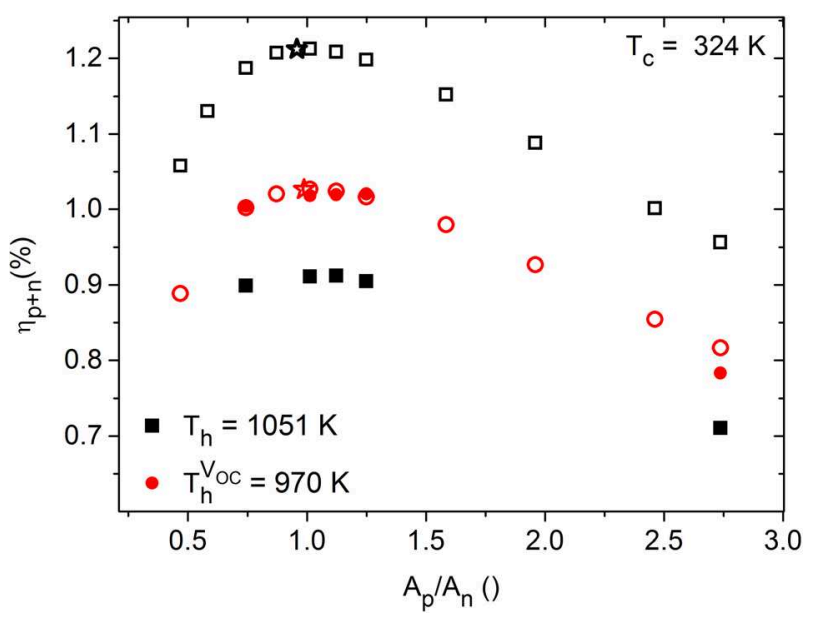

FIG. 6. Experimental (filled) and theoretical converter efficiency (open symbols) for the fabricated and additional $A_{p} / A_{n}$ ratios for $T_{c}=324 \mathrm{~K}$. For the calculation of the heat flow and the efficiency, either the measured $T_{h}$ or the estimated temperature $T_{h}^{V O C}$ was used. For both temperatures, $\left(A_{p} / A_{n}\right)^{*}$ is shown (stars). As radiative losses are neglected, $\eta_{p+n}$ is overestimated by about $8 \%$ (cf. Section III in supplementary material ${ }^{34}$ ). 
For the presented material combination, the most efficient ratio is close to $A_{p} / A_{n}=1$, but for material combinations with very different properties, this commonly used ratio may result in a tremendous decrease of the efficiency. Thus, a fast calculation of the most efficient ratio according to Eq. (2) is highly recommended prior to generator fabrication. Furthermore, around the maximum efficiency the curve is relatively flat (Figure 6). This offers the opportunity for cost weight or volume reduction without remarkable efficiency losses, which is of particular importance for mobile applications, e.g., in the automotive industry.

Finally, the long-term stability of the generator with $A_{p} / A_{n}=1.12$ is investigated for $114 \mathrm{~h}$ (Figure 7) for the final application on a casting furnace at vonRoll casting. The test is performed at $T_{h} \approx 1160 \mathrm{~K}$ and $T_{c} \approx 355 \mathrm{~K}$. Although $T_{h}$ is close to the decomposition temperature $(1200 \mathrm{~K})$ of the cobalt oxide ${ }^{27}$ and the melting point of silver $(1235 \mathrm{~K})$, no degradation effects are observed. The internal resistance $R_{i}$ of the generator is constant (Figure 7(d)), which points out the long time stability of the Ag-contacts achieved by intermixing with the oxide powder. The maximum power output reaches a peak value of $237 \mathrm{~mW}$ and varies by about $2 \%$. This variation is probably induced by the $3 \%$ variation of $\Delta \mathrm{T}$, which also reflects in a change of $V_{O C}$. Normalization of the maximum power output to the total cross-sectional area of the legs, the leg volume, and the mass of the thermoelectric legs lead to values of $0.58 \mathrm{~W} / \mathrm{cm}^{2}, 1.4 \mathrm{~W} / \mathrm{cm}^{3}$, and $0.31 \mathrm{~W} / \mathrm{g}$, respectively. This power corresponds to a maximum efficiency of $0.99 \%$ for the measured $T_{h}$ and $1.08 \%$ if the more reliable estimated $T_{h}^{V_{O C}}=1085 \mathrm{~K}$ is used for the calculation of the heat flow.

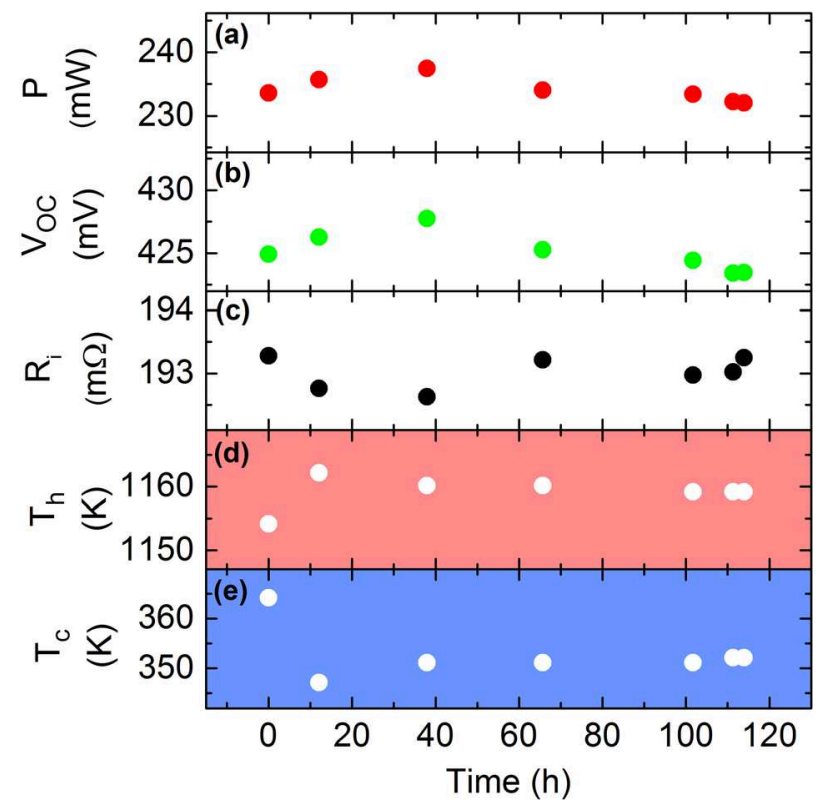

FIG. 7. Stability of the generator with $A_{p} / A_{n}=1.12$ during $114 \mathrm{~h}$ of energy conversion. The maximum power output (a), the open circuit voltage (b), and the internal resistance (c) are constant. Small variations might result from small drifts of the hot (d) and the cold side temperature (e).

\section{CONCLUSION}

A series of four-leg thermoelectric modules with varying $A_{p} / A_{n}$ ratio was fabricated, characterized, and successfully tested in long-term tests at very high temperatures. It was shown that the power output and the efficiency of the thermoelectric generators increase strongly for an adjustment of the cross section ratio $A_{p} / A_{n}$ according to the compatibility approach. For $A_{p} / A_{n}$ differing strongly from $\left(A_{p} / A_{n}\right)^{*}$, a relative decrease of the experimental efficiency of up to $23 \%$ was observed. For the best performing module with $A_{p} / A_{n}=$ 1.12 , the long-term thermal stability was demonstrated, which highlights the reliability and the potential of the oxide thermoelectric converters for high temperature applications. The measured maximum power output, the maximum power density normalized to the total cross-sectional area of the legs, the leg volume, and the mass of the thermoelectric legs were found to be $237 \mathrm{~mW}, 0.58 \mathrm{~W} / \mathrm{cm}^{2}, 1.4 \mathrm{~W} / \mathrm{cm}^{3}$, and $0.31 \mathrm{~W} / \mathrm{g}$, respectively. Based on the measured temperature $T_{h}=1160 \mathrm{~K}$ and the smaller estimated $T_{h}^{V O C}=1085 \mathrm{~K}$, the calculated heat flow leads to efficiencies of $0.99 \%$ and $1.08 \%$, respectively. Theoretically, even higher efficiencies are possible since for $\mathrm{Ca}_{3} \mathrm{Co}_{3.9} \mathrm{O}_{9.3+\delta}$ higher $\mathrm{ZT}$ were reported in literature.

Despite its simplifications, the theoretical compatibility model describes the experimental trends very well and offers an easy way for the improvement of thermoelectric converters. Especially, the calculated dependency of the efficiency on the $A_{p} / A_{n}$ ratio can be used for cost, weight, and volume reduction, which is crucial for large-scale applications of thermoelectric converters for waste heat recovery.

\section{ACKNOWLEDGMENTS}

Funding from the Bundesamt für Energie (BFE) under Grant No. Si/500601, from vonRoll and from the Competence Centre Energy and Mobility (HITTEC Project) are greatly acknowledged. Furthermore, the authors want to thank Yoko Matsumura and Tomoyuki Urata for support with the module fabrication, Roman Furrer for the determination of the emissivities and Matthias Schmid for fruitful discussions.

${ }^{1}$ W. Brostow, T. Datashvili, H. E. H. Lobland, T. Hilbig, L. Su, C. Vinado, and J. White, J. Mater. Res. 27, 2930 (2012).

${ }^{2}$ J. Leszczynski, K. T. Wojciechowski, and A. L. Malecki, J. Therm. Anal. Calorim. 105, 211 (2011).

${ }^{3}$ D. Zhao, C. Tian, S. Tang, Y. Liu, and L. Chen, J. Alloys Compd. 504, 552 (2010).

${ }^{4}$ K. Gałązka, S. Populoh, L. Sagarna, L. Karvonen, W. Xie, A. Beni, P. Schmutz, J. Hulliger, and A. Weidenkaff, Phys. Status Solidi A 211, 1259 (2014).

${ }^{5}$ S. Populoh, O. C. Brunko, K. Gałązka, W. Xie, and A. Weidenkaff, Materials 6, 1326 (2013).

${ }^{6}$ A. Weidenkaff, M. H. Aguirre, L. Bocher, M. Trottmann, P. Tomes, and R. Robert, J. Korean Ceram. Soc. 47, 47 (2010).

${ }^{7}$ S.-M. Choi, K.-H. Lee, C.-H. Lim, and W.-S. Seo, Energy Convers. Manage. 52, 335 (2011).

${ }^{8}$ R. Funahashi, S. Urata, K. Mizuno, T. Kouuchi, and M. Mikami, Appl. Phys. Lett. 85, 1036 (2004).

${ }^{9}$ A. Inagoya, D. Sawaki, Y. Horiuchi, S. Urata, R. Funahashi, and I. Terasaki, J. Appl. Phys. 110, 123712 (2011).

${ }^{10}$ C.-H. Lim, S.-M. Choi, W.-S. Seo, and H.-H. Park, J. Electron. Mater. 41, 1247-1255 (2012). 
${ }^{11}$ P. Mele, H. Kamei, H. Yasumune, K. Matsumoto, and K. Miyazaki, Met. Mater. Int. 20, 389-397 (2014).

${ }^{12}$ E. S. Reddy, J. G. Noudem, S. Hebert, and C. Goupil, J. Phys. D: Appl. Phys. 38, 3751 (2005).

${ }^{13}$ P. Tomeš, M. Trottmann, C. Suter, M. H. Aguirre, A. Steinfeld, P. Haueter, and A. Weidenkaff, Materials 3, 2801 (2010).

${ }^{14}$ R. Funahashi, M. Mikami, T. Mihara, S. Urata, and N. Ando, J. Appl. Phys. 99, 066117 (2006).

${ }^{15}$ R. Funahashi and S. Urata, Int. J. Appl. Ceram. Technol. 4, 297 (2007).

${ }^{16}$ C.-H. Lim, S.-M. Choi, W.-S. Seo, and H.-H. Park, J. Electron. Mater. 41, 1247 (2011).

${ }^{17}$ I. Matsubara, R. Funahashi, T. Takeuchi, S. Sodeoka, T. Shimizu, and K. Ueno, Appl. Phys. Lett. 78, 3627 (2001).

${ }^{18}$ J. G. Noudem, S. Lemonnier, M. Prevel, E. S. Reddy, E. Guilmeau, and C. Goupil, J. Eur. Ceram. Soc. 28, 41 (2008).

${ }^{19}$ S. Urata, R. Funahashi, T. Mihara, A. Kosuga, S. Sodeoka, and T. Tanaka, Int. J. Appl. Ceram. Technol. 4, 535 (2007).

${ }^{20}$ R. Funahashi, Sci. Adv. Mater. 3, 682 (2011).

${ }^{21}$ S. Populoh, M. Trottmann, O. C. Brunko, P. Thiel, and A. Weidenkaff, Funct. Mater. Lett. 06, 1340012 (2013).

${ }^{22} \mathrm{P}$. Tomeš, C. Suter, M. Trottmann, A. Steinfeld, and A. Weidenkaff, J. Mater. Res. 26, 1975 (2011).

${ }^{23}$ T. Hung, Segmented Thermoelectric Oxide-Based Module (Technical University of Denmark, 2014).

${ }^{24}$ L. T. Hung, N. Van Nong, G. J. Snyder, M. H. Viet, B. Balke, L. Han, E. Stamate, S. Linderoth, and N. Pryds, Energy Convers. Manage. 99, 20 (2015).

${ }^{25}$ G. Snyder and T. Ursell, Phys. Rev. Lett. 91, 148301 (2003).
${ }^{26}$ D. Crane, J. LaGrandeur, V. Jovovic, M. Ranalli, M. Adldinger, E. Poliquin, J. Dean, D. Kossakovski, B. Mazar, and C. Maranville, J. Electron. Mater. 42, 1582 (2013).

${ }^{27}$ E. Woermann and A. Muan, J. Inorg. Nucl. Chem. 32, 1455 (1970).

${ }^{28}$ J. W. Fergus, J. Eur. Ceram. Soc. 32, 525 (2012).

${ }^{29}$ P. Thiel, J. Eilertsen, S. Populoh, G. Saucke, M. Döbeli, A. Shkabko, L. Sagarna, L. Karvonen, and A. Weidenkaff, J. Appl. Phys. 114, 243707 (2013).

${ }^{30}$ G. J. Snyder, Thermoelectrics Handbook: Macro to Nano (Taylor \& Francis/CRC Press, Boca Raton, 2006), Chap. 9.

${ }^{31}$ L. L. Baranowski, G. J. Snyder, and E. S. Toberer, J. Appl. Phys. 113, 204904 (2013).

${ }^{32}$ W. Wijesekara and L. Rosendahl, Phys. Status Solidi A 212, 591 (2015).

${ }^{33}$ W. H. Clingman, Adv. Energy Convers. 1, 61 (1961).

${ }^{34}$ See supplementary material at http://dx.doi.org/10.1063/1.4926476 for detailed information about the determination of the power output at the same open circuit voltage, the change of the converter resistance, and the estimation of radiative losses.

${ }^{35}$ A. C. Masset, C. Michel, A. Maignan, M. Hervieu, O. Toulemonde, F. Studer, B. Raveau, and J. Hejtmanek, Phys. Rev. B 62, 166 (2000).

${ }^{36}$ G. Saucke, S. Populoh, N. Vogel-Schäuble, L. Sagarna, K. Mogare, L. Karvonen, and A. Weidenkaff, MRS Proc. 1543, 83 (2013).

${ }^{37}$ S. Populoh, M. H. Aguirre, O. C. Brunko, K. Galazka, Y. Lu, and A. Weidenkaff, Scr. Mater. 66, 1073 (2012).

${ }^{38}$ K. Bartholomé, B. Balke, D. Zuckermann, M. Köhne, M. Müller, K. Tarantik, and J. König, J. Electron. Mater. 43, 1775 (2014).

${ }^{39}$ W. Seifert, K. Zabrocki, G. J. Snyder, and E. Müller, Phys. Status Solidi A 207, 760 (2010). 